\title{
A Data-Driven Method for Variation in Animated Smiles (Extended Abstract)
}

\author{
Nick Sohre, Stephen J. Guy \\ University of Minnesota Department of Computer Science and Engineering \\ (sohre, sjguy)@cs.umn.edu
}

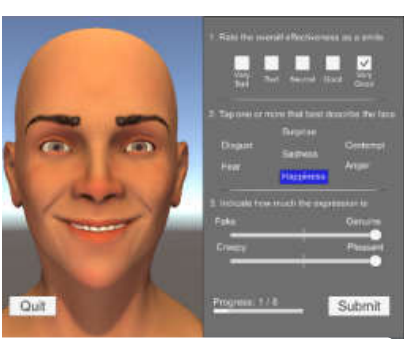

User Study

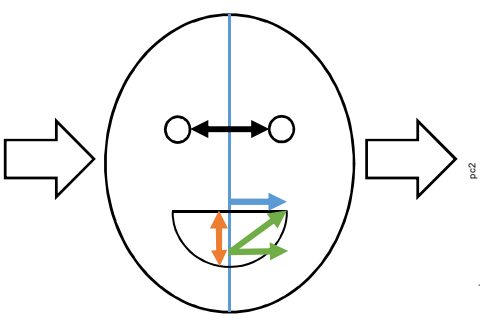

Facial Space

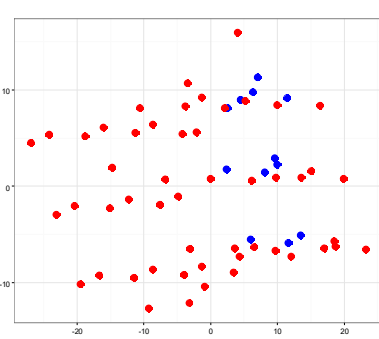

Non-Parametric Classifier

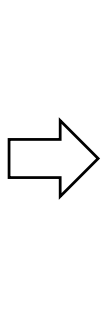

\& Blendshape Solve

Figure 1: A graphical overview of our method. We systematically explore plausible faces in blendshape space, and annotate them with perceived level of happiness via a large scale user study. We then parameterize the faces into a new space based on facial features, and feed them into a non-parametric classifier. New smiles are generated by sampling new faces in this space, keeping those predicted to be happy.

\begin{abstract}
Animated digital characters play an important role in virtual experiences. In this work, we utilize data from a large scale user study as training data for a generative model for producing a variety of animated smiles. Our method involves a four stage process that samples a variety of facial expressions, and annotates them with perceived happiness from the user study. The expressions are then transformed into a standardized space and used by a non-parametric classifier to predict happiness of new smiles.
\end{abstract}

Keywords: data-driven facial animation, digital character emotion, computer graphics

Concepts: •Computing methodologies $\rightarrow$ Animation; Modeling and simulation;

\section{Intro and Background}

Animating digital characters has an important role in computer assisted experiences, from video games to movies to interactive robotics. A critical component of creating compelling interactions is the representation and control of the human face. Previous work has shown that a variety of character appearances is important in creating immersive experiences [McDonnell et al. 2008; OSullivan 2009], and that the human faces is of particular importance [Sinha et al. 2006]. In this work, we utilize user-study data to inform a generative process for varied animated smiles. Through the formulation of a standard space for facial expressions, a large scale user study capturing perceived happiness, and a data-driven method for producing new smiles, we are able to generate faces that exhibit a variety of happy smiles. This diversity can allow rich interactions

Permission to make digital or hard copies of part or all of this work for personal or classroom use is granted without fee provided that copies are not made or distributed for profit or commercial advantage and that copies bear this notice and the full citation on the first page. Copyrights for thirdparty components of this work must be honored. For all other uses, contact the owner/author(s). (c) 2016 Copyright held by the owner/author(s).

MiG '16, October 10-12, 2016, Burlingame, CA, USA

ISBN: 978-1-4503-4592-7/16/10

DOI: http://dx.doi.org/10.1145/2994258.2994290 with digital characters to be built automatically, overcoming limitations of current practices.

Current methods for producing facial expressions tend towards utilizing facial performance capture, physically based models of facial movement, or using animations handcrafted by artists. Models based on physical interactions of skin, subcutaneous tissue, muscle, and skeletal structure [Waters 1987; Cong et al. 2015; Lee et al. 1995] can achieve very realistic behavior, at the cost of a high level of complexity in the model. Approaches that focus on constructing detailed, realistic facial models from depth $\&$ reflectance sensor data [Pighin et al. 2006; Li et al. 2013] can be limited to the expressions produceable by human actors, and may require calibration or sophisticated hardware.

\section{Method}

Our approach involves a multi-step process that uses a paramaterized 3D model of the human face, annotated training data, and a non-parametric classifier.

\subsection{Facial Space}

We begin by representing the human face and its plausible deformations via a base mesh and a set of interpolative blendshapes. We then define a parameter space based on key feature points of the face, called facial space, and establish a method for traversing between this space and interpolative blendshape weights. This allows for our method to extend to different base meshes and arbitrary sets of blendshapes. Specifically, we focus this space around the movements of the mouth. The impetus for this is multi-faceted; the mouth is a crucial (if not the defining) element in a smile, exhibits well-known structure for identifying key feature points, and allows our work to easily extend to collaborations with professionals in facial reconstructive surgery. The resulting space is defined as several key relationships between the mouth corners and centers of the upper and lower lips, as identified by medical professionals. These include the angle formed by the mouth corner and resting lip position, mouth center, lip separation (dental show), and mouth corner distance to the saggital plane (extent). 


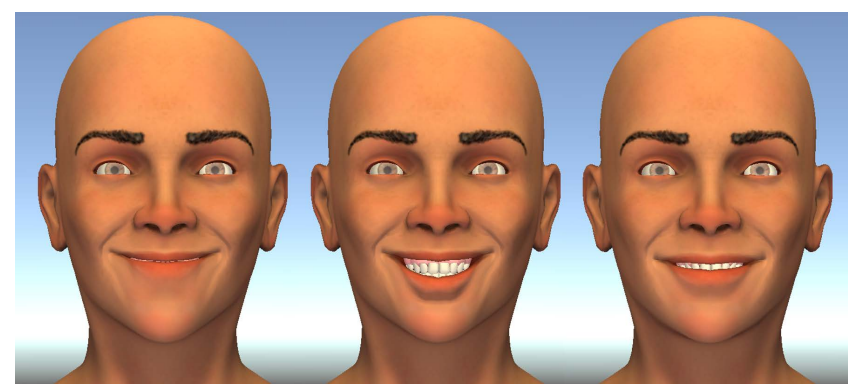

Figure 2: A variety of happy faces generated by our method.

\subsection{Data Collection}

We take as training input facial expressions labeled by the perceived happiness of the smile. To produce this data, we performed a systematic sweep of facial expressions in blendshape space, and produced animated renderings of each on our digital human facial model. We then conducted a large scale user study to label the data with perceived smile effectiveness. Participants in the user study were shown several smiles on a tablet device, and asked to assess each in terms of the overall happiness of the face and effectiveness of the smile. The user study was conducted at the 2015 Minnesota State Fair, where over 10,000 assessments were collected from over 900 participants.

\subsection{Smile Generation}

We then built a non-parametric classifier on the training data to predict, given a new point in facial space, the perceived happiness in the resulting expression. To generate new smiles, points in facial space are sampled until one is found within the desired range of predicted happiness. These can then be converted into blendshape space to produce the resulting facial expressions.

\section{Results}

Using our method we are able to generate a variety of happy smiles. Figure 2 demonstrates examples with varying positions in facial space (different angles, extents, and dental show). Although the smile generation takes place in facial space, which only concerns configurations of the mouth, our method is still able to produce a variety of happy faces. Overall, the runtime complexity of our system is $O\left(m b+n^{2}\right)$, where $m$ is the dimensionality of facial space, $n$ is the number of training samples, and $b$ is the number of blendshapes. As a consequence, our method can scale well with increasing blendshapes or increasing facial space dimensions (though not necessarily both).

In the future, we plan to explore the generation of faces with different emotions and emotional mixtures, extend facial space to include other regions of the face, and perform a more robust analysis of the machine learning used to predict the perceived emotional intent of points in facial space.

\section{Acknowledgements}

This work has been supported in part by the National Science Foundation through grant \#CHS-1526693

\section{References}

Cong, M., Bao, M., Bhat, K. S., Fedkiw, R., et Al. 2015. Fully automatic generation of anatomical face simulation models. In Proceedings of the 14th ACM SIGGRAPH/Eurographics Symposium on Computer Animation, ACM, 175-183.

Lee, Y., Terzopoulos, D., And Waters, K. 1995. Realistic modeling for facial animation. In Proceedings of the 22nd annual conference on Computer graphics and interactive techniques, ACM, 55-62.

LI, H., Yu, J., Ye, Y., AND BREgler, C. 2013. Realtime facial animation with on-the-fly correctives. ACM Trans. Graph. 32, 4, $42-1$.

McDonnell, R., Larkin, M., Dobbyn, S., Collins, S., AND O'SULlivan, C. 2008. Clone attack! perception of crowd variety. In ACM Transactions on Graphics (TOG), vol. 27, ACM, 26.

OSullivan, C. 2009. Variety is the spice of (virtual) life. In International Workshop on Motion in Games, Springer, 84-93.

Pighin, F., Hecker, J., Lischinski, D., Szeliski, R., AND SALESIN, D. H. 2006. Synthesizing realistic facial expressions from photographs. In ACM SIGGRAPH 2006 Courses, ACM, 19.

Sinha, P., BAlas, B., Ostrovsky, Y., AND Russell, R. 2006. Face recognition by humans: Nineteen results all computer vision researchers should know about. Proceedings of the IEEE 94, 11, 1948-1962.

WATERS, K. 1987. A muscle model for animation threedimensional facial expression. In Proceedings of the 14th Annual Conference on Computer Graphics and Interactive Techniques, ACM, New York, NY, USA, SIGGRAPH '87, 17-24. 